\title{
Las lesiones de la sustancia blanca cerebral y la retinopatía se asociaron a una mayor incidencia de ACV
}

Cerebral White Matter Lesions, Retinopathy,and Incident Clinical Stroke. Wong T, Klein R, Sarret A y col. JAMA 2002; 288:67-74.

\section{Objetivo}

Examinar la relación entre las lesiones de la sustancia blanca cerebral (LSBC), las anormalidades de la microvasculatura de la retina, y la incidencia de accidente cerebrovascular (ACV) clínico en personas sanas de edad media.

\section{Diseño}

Estudio de cohorte prospectivo* ARIC (Atherosclerosis Risk in Communities Study), iniciado en 1987-1989.

\section{Lugar}

Cuatro comunidades de EE.UU.

\section{Pacientes}

Se incluyeron 1.684 personas entre 51 y 72 años, a quienes se les realizó una resonancia nuclear magnética (RNM) y una retinografía en el tercer examen (1993-1995) del estudio.

\section{Evaluación de factores pronósticos}

Se evaluó la presencia de lesiones en la sustancia blanca cerebral y de cambios vasculares retiniananos. Esto fue realizado por dos operadores expertos, ciegos respecto a las condiciones clínicas de los participantes y de los hallazgos del resto de los estudios complementarios.

\section{Medición de Resultados Principales}

Presencia de LSBC, definida por métodos estandarizados de RNM y la presencia o ausencia de anormalidades específicas de la mi- crovasculatura de la retina. El desenlace principal fue la incidencia de ACV clínico, luego de un seguimiento medio de 4,7 años, de acuerdo a la presencia o ausencia de LSBC y retinopatía. Se reporta el análisis multivariado*, ajustado por edad, sexo, raza y factores de riesgo vascular.

\section{Resultados Principales}

Las personas con retinopatía tuvieron mayores probabilidades de desarrollar LSBC que aquellas sin retinopatía (22,9\% vs. $9,9 \%$, Odds Ratio ajustado [OR] 2,5;IC95\% 1,5 a 4,0).

La incidencia acumulativa de ACV a los cinco años fue mayor en las personas con LSBC, que aquellas que no las tuvieron $(6,8 \%$ vs. $1,4 \%$, RR ajustado 3,4 ; IC95\% 1,5 a 7,7 ), y en personas con retinopatía en relación a aquellas que no la tenían ( $8,0 \%$ vs. $1,4 \%$, RR ajustado 4,9; IC95\% 2,0 a 11,9).

Las personas con LSBC y retinopatía, tuvieron una incidencia acumulativa de ACV significativamente más elevada a los 5 años que aquellas que no tenían ninguna de ellas ( $20 \%$ vs. $1,14 \%$, RR ajustado 18,$1 ;$ IC $95 \% 5,9$ a 55,4 ).

\section{Conclusiones}

Las personas con lesiones en la sustancia blanca cerebral tuvieron mayores probabilidades de tener anormalidades de la microvasculatura de la retina y de tener a la vez un riesgo incrementado de desarrollar un ACV.EI riesgo fue mayor cuando las lesiones en la sustancia blanca cerebral se hallaban presentes en personas con retinopatía.

\section{Comentario}

Hasta el $80 \%$ de la población mayor de 65 años puede tener cambios en la sustancia blanca cerebral en la RNM ${ }^{1}$.Es este un problema frecuente con el que se enfrentan los médicos en su práctica habitual.En particular porque en muchas oportunidades su hallazgo no se relaciona con el problema que motivó la solicitud del estudio.La patogénesis de estas lesiones, así como su implicancia clínica, han sido motivo de controversia a lo largo de los años. Varios estudios histopatológicos relacionan estos cambios de la sustancia blanca con complicaciones isquémicas secundarias a enfermedad de la microvasculatura cerebral ${ }^{2}$.

Los autores de este trabajo se basan en la hipótesis largamente planteada ${ }^{3}$, que dada la similitud entre la anatomía, la fisiología y la embriología de las ar teriolas retinianas con las cerebrales;cambios en la microvasculatura de la retina reflejarían a su vez cambios en la del encéfalo.De esta manera, se podría determinar qué pacientes se hallarían en mayor riesgo de sufrir una patología cerebrovascular.

Los autores afirman haber demostrado que las lesiones de la sustancia blanca cerebral se asocian en forma independiente con el riesgo de padecer un ACV. Como dato adicional, si a dichas lesiones se les asocia una retinopatía, la probabilidad de sufrir un ACV en los 5 años subsiguientes aumentaría 18 veces respecto a la población sana.
Debe hacerse notar una importante limitación (señalada por los propios autores):la hipertensión es un factor de riesgo común, tanto para las LSBC, como para las alteraciones de la microvasculatura retiniana, y para el ACV. A pesar de que los autores controlan por los valores de presión, este fenómeno puede llevar a sobrevalorar en alguna medida la magnitud de la asociación.

También sostienen que estos hallazgos pueden tener importantes implicancias clínicas.Sin embargo, en este punto no compartimos su excesivo entusiasmo.En la práctica clínica estos pacientes con LSBC en la RMN, ya sea con o sin retinopatía, ya son tratados en forma rutinaria para prevenir un ACV, debido a que esta asociación entre LSBC y ACV, por lo comentado en el primer párrafo, es aceptada como tal desde tiempo atrás por la mayoría de los especialistas en patología cerebrovascular.Sin embargo no se puede negar la significancia de haberse logrado cuantificar la magnitud de este riesgo.

Conclusión del comentador: A pesar de no modificar la práctica habitual de tratar a los pacientes con LSBC o retinopatía para la prevención del ACV, este estudio poblacional logra cuantificar la magnitud del riesgo que confieren (LSBC:tres veces más ACV, retinopatía: cinco veces más; ambas: casi veinte veces más).

\section{Dr. Diego Bauso [ Servicio de Neurología.Hospital Italiano de Buenos Aires ]}

\section{Referencias}

1.Ylikkoski A, Erkinjuntti T, Raininko R, et al.White matter hyperintensities on MRI in the neurologically nondiseased elderluy: analysis of cohorts of consecutive subjects aged 55 to 85 years living at home. Stroke 1995;26:1171-1177.

2. Fazekas F, Kleinert R, Offarbacher H, et al.Pathologic correlation of incidental MRI white matter signal hyperintensities.Neurology 1993; 43:1683-1689.

3. Goto I, Kimoto K, Katsuki S, et al.Pathological studies on the intracerebral and retinal arteries in cerebrovascular and noncerebrovascular diseases. Stroke 1975;6:263-269. 